\title{
On the Age, Spectral Type, Orbit, and Comparison to Evolutionary Models of AB Dor C
}

\author{
L. M. Close ${ }^{1}$, E. L. Nielsen ${ }^{1}$, J. C. Guirado ${ }^{2}$, B. A. Biller ${ }^{1}$, R. \\ Lenzen $^{3}$, W. Brandner ${ }^{3}$, M. Hartung ${ }^{4}$ and C. Lidman ${ }^{4}$ \\ ${ }^{1}$ Steward Observatory, University of Arizona, 933 N. Cherry Ave, Tucson, AZ 85721 USA, \\ email: lclose@as.arizona.edu \\ ${ }^{2}$ Departament d'Astronomia i Astrofisica, Universitat de Valencia, E-46100 Burjassot, \\ Valencia, Spain \\ ${ }^{3}$ Max-Plank-Institut für Astronomie, Königstuhl 17, D-69117 Heidelberg, Germany \\ ${ }^{4}$ European Southern Observatory, Alonso de Cordova 3107, Santiago 19, Chile
}

\begin{abstract}
We expand upon the results of Close et al. 2005 regarding the young, low-mass object $\mathrm{AB}$ Dor $\mathrm{C}$ and its role as a calibration point for theoretical tracks. We argue for a new $70 \pm 30$ Myr age estimate and present two additional detections of $\mathrm{C}$ with the Simultaneous Differential Imaging (SDI) camera. Our improved analysis (Nielsen et al. 2005) confirms our spectral type of M8 $( \pm 1)$ and mass of $0.090 \pm 0.003 \mathrm{M}_{\text {sun }}$ for AB Dor C. However, Luhman \& Potter (2006) argue for a hotter spectral type $(\mathrm{M} 6 \pm 1)$. Here we adopt the consistent spectral range of $\mathrm{M} 7 \pm 1$ in this paper as a final spectral type. Plotting AB Dor C (and all other young $(<0.12 \mathrm{Myr}$ ), lowmass $\left(0.3-0.05 M_{\text {sun }}\right)$ objects with accurate dynamical masses) on the HR diagram suggests a trend where current evolutionary models tend to over-predict the temperature (or under-predict the mass) for young low-mass stars and high-mass brown dwarfs. With our uncertainties, there is a $\sim 90 \%$ chance that the mass of $\mathrm{AB}$ Dor $\mathrm{C}$ is underestimated by the DUSTY tracks in the HR diagram.
\end{abstract}

Keywords. Infrared: Stars, Stars: Formation, Stars: Low-Mass, Brown Dwarfs, Stars: Pre-MainSequence, Stars: Individual: AB Dor.

\section{Introduction}

The study of young, low-mass objects has been yielding increasingly fruitful science, yet the field remains dependent on evolutionary models to properly interpret the data that are collected from these objects. In particular, mass, while a fundamental property, is very rarely measured directly, and instead must be inferred from theoretical tracks (e.g., Burrows, Sudarsky, and Lunine 2003, Chabrier et al. 2000). It is thus of great interest to find calibrating objects that can link a dynamically measured mass with observables such as NIR (1-2 $\mu \mathrm{m})$ fluxes and spectral types.

In our previous work (Close et al. 2005), we reported the first direct detection of the low-mass companion to the young star AB Dor A (separation $0.15^{\prime \prime}, \Delta \mathrm{H}=5.2 \mathrm{mag}$ ), along with measurements of the JHKs fluxes, spectral type, and dynamically determined mass of AB Dor C. Upon comparing these results with the predictions of Chabrier et al. 2000, we found the models to be systematically over-predicting the fluxes and temperature of $\mathrm{AB}$ Dor $\mathrm{C}$, given an age of the system of $50 \mathrm{Myr}$. Put another way, the model masses seem to be underestimating the mass of a low-mass object given its age, NIR fluxes, and spectral type. Since the publication of these results, another calibrating object has been reported by Reiners, Basri, and Mohanty 2005: USco CTIO 5. While this equal-mass binary is younger $(\sim 8 \mathrm{Myr})$ and more massive (total $\operatorname{mass}_{A} \geqslant 0.32 \mathrm{M}_{\text {sun }}$ ) than $\mathrm{AB}$ Dor 


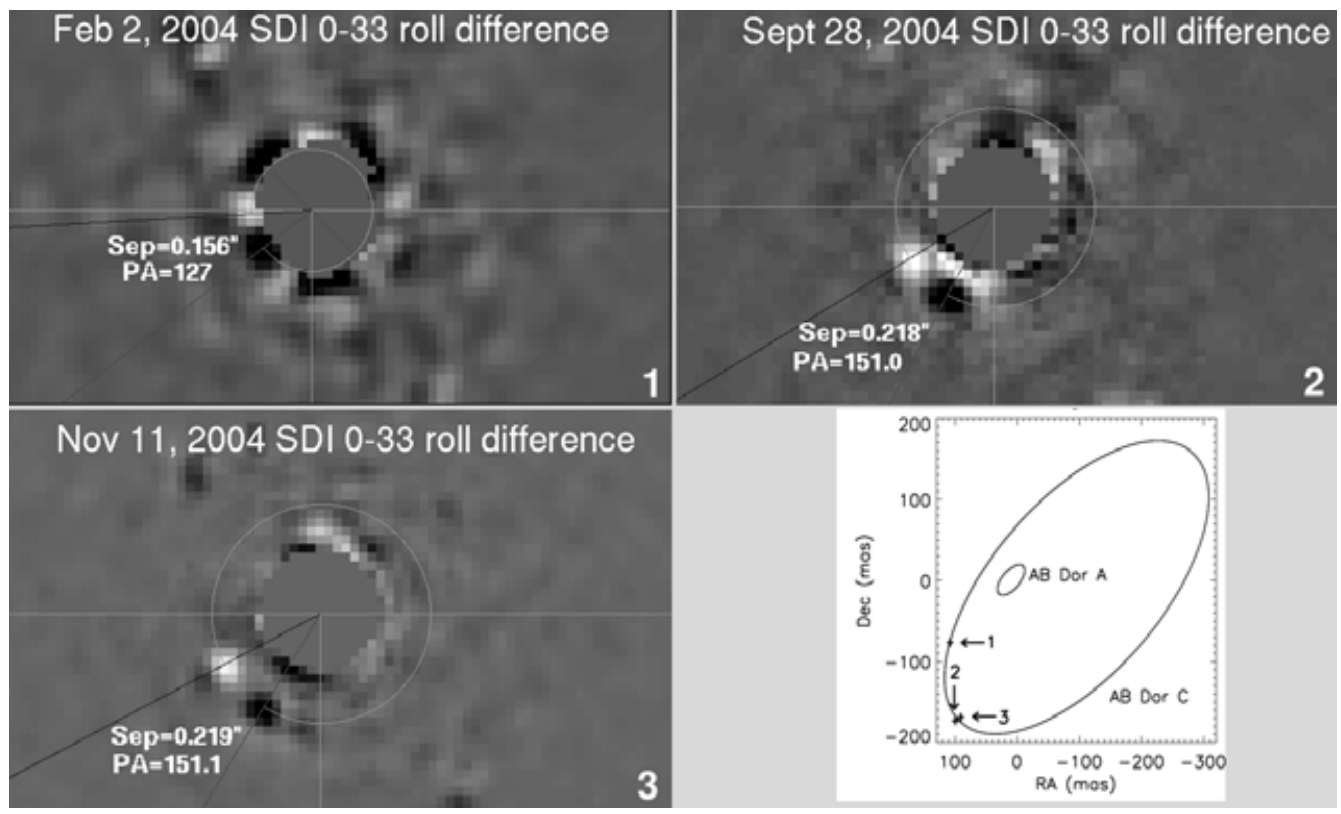

Figure 1. The different epochs of AB Dor C measured during 2004 with the VLT SDI device (from Nielsen et al. 2005). Each of the numbered panels shows an individual epoch of SDI observations, with images at position angles of $0^{\circ}$ and $33^{\circ}$ subtracted from each other, showing a positive and negative signal from AB Dor C. The inner pixels of AB Dor A have been intentionally saturated, and have been removed from the image. The orbital motion of the companion can clearly be seen over this span of time. The bottom-right panel shows these locations against a plot of the full orbit of $\mathrm{AB}$ Dor C. The 11 additional VLBI/Hipparcos measurements of the reflex motion of $\mathrm{AB}$ Dor $\mathrm{A}$ (which went into finding the orbital solution) are not shown on this plot (See Close et al. 2005).

$\mathrm{C}$, Reiners et al. find the same trend of models under-predicting masses based simply on photometric and spectroscopic data applied to the HR diagram. A similar trend for such masses was previously noted by Hillenbrand and White 2004. Moreover, this trend has been theoretically predicted for higher masses by Mohanty, Jayawardhana, and Basri 2004, and by Marley et al. 2005 for planetary masses.

In this paper, we seek to expand on our earlier results from Close et al. 2005 and Nielsen et al. 2005, using an improved spectral reduction. We also present an improved orbital fit based on additional astrometric data from Nielsen et al. (2005), as well we address concerns raised by Luhman, Stauffer, and Mamajek 2005 regarding the age of the AB Dor system. In addition we incorporate the new spectra type determination of Luhman \& Potter (2006) into our analysis.

\section{An Improved Spectral Reduction}

As described in Close et al. 2005, in February 2004 we obtained 20 minutes of K-band spectra using the Very Large Telescope (VLT), following our initial detection of AB Dor C.

Improved spectra from that of Close et al. were re-reduced by Nielsen et al. 2005. The spectra for AB Dor C is shown in Figure 2 from Nielsen et al. 2005. All of the templates used in Figure 2 are significantly younger than AB Dor C. To properly bound the spectral 

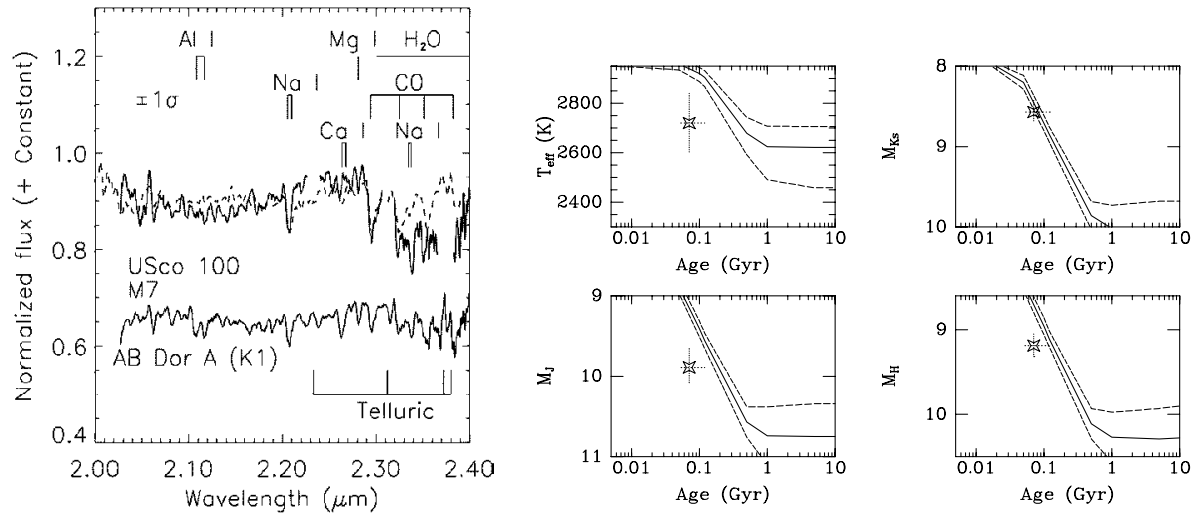

Figure 2. LEFT: The spectrum of AB Dor C shown against a number of young ( 10 Myr), low-surface gravity objects. RIGHT: old field objects. The features seem to be bound between the M7 and M9.5 templates. From Nielsen et al. 2005.

type, we also compare to field objects, as we did in Nelsen et al. 2005. The spectrum seems to fit best in the sequence between an M7 and an M9.

Recently (after the IAUC 200 conference) an independent reduction of our dataset by Luhman \& Potter (2006) finds an earlier spectral type M6 \pm 1 for AB Dor C when comparing to old M dwarfs. A spectral type of M7 is consistent with all AB Dor C spectral and photometric studies. Since these studies used independent approaches to the data reduction it is reasonable to adopt a final spectral type of $M 7 \pm 1$ which is consistent with all the colors and spectral types of Close et al. (2005), Nielsen et al. (2005), and Luhman \& Potter (2006) measured for AB Dor C to date.

\section{Discussion}

\section{Improved Orbit}

Our earlier paper (Close et al. 2005) was based on observations conducted at the VLT in February of 2004. Since this work was published, we have reduced additional Simultaneous Differential Imaging (SDI, see Close et al. 2003; Lenzen et al. 2004) data from September and November of 2004. While data through the narrow-band SDI filters do not provide us with any improved photometric information (beyond confirmation that between $\mathrm{AB}$ Dor $\mathrm{A}$ and $\mathrm{C}, \Delta \mathrm{H}=5.20)$, these images do give us additional astrometric data points, allowing us to refine the orbit. Figure 1 shows each epoch of measuring the position of $\mathrm{AB}$ Dor $\mathrm{C}$ with respect to $\mathrm{A}$.

The most immediate consequence of the fit of Nielsen et al. 2005 is that the mass of AB Dor $\mathrm{C}$ remains at $0.090 \mathrm{M}_{\text {sun }}$. As reported in Guirado et al. 2005, we notice that the error bars shrink from $0.005 \mathrm{M}_{\text {sun }}$ to $0.003 \mathrm{M}_{\text {sun }}$. This confidence with which we know the mass of $\mathrm{AB}$ Dor $\mathrm{C}$ makes it an ideal object for calibrating theoretical evolutionary tracks.

\subsection{The Age of $A B$ Dor}

In Close et al. 2005 it was argued that due to the excess luminosity of AB Dor A and C compared to the Pleiades, and A's large $\sim 300 \mathrm{~mA} \mathrm{Li} \mathrm{equivalent} \mathrm{width} \mathrm{and} \mathrm{very} \mathrm{fast}$ $(12.3 \mathrm{~h})$ rotation, that the age of the system was 30-100 Myr. An age of $50(-20,+50)$ Myr was adopted, which was consistent with the 50 Myr published age of the AB Dor moving group (Zuckerman, Song, and Bessell 2004). Recently Luhman et al. 2005 has 

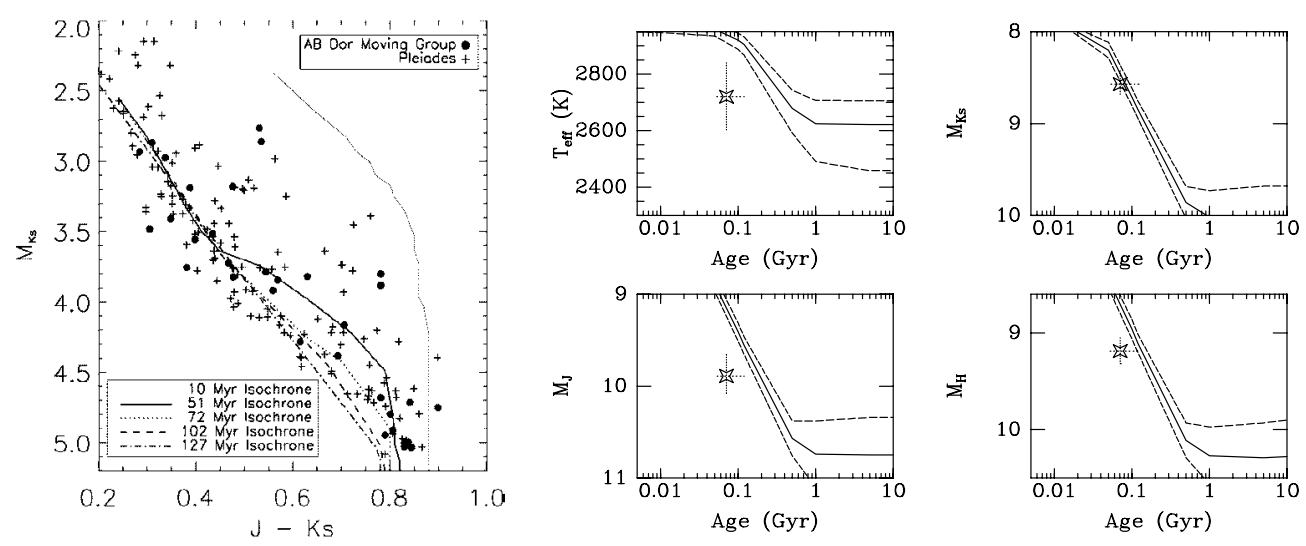

Figure 3. LEFT: A NIR color-magnitude diagram of medium-mass members of the Pleiades (compiled from the literature) and the AB Dor moving group (Zuckerman et al. 2004), with the theoretical isochrones of Baraffe et al. 1998. The offset between the single star locus of the two groups redward of J-Ks $~ 0.4$ suggests a younger age for the AB Dor moving group, closer to 70 Myr. RIGHT: A comparison between our Close et al. (2005) photometry and effective temperature to the dusty models for the 0.085-0.095 mass tracks appropriate of AB Dor C. Ages of $70 \pm 30 \mathrm{Myr}$ are adopted for AB Dor C. Note how the models overestimate the values observed. The J, H and Ks fluxes of Luhman \& Potter (2006) agree with these values; however, their uncertainties are higher and they adopt an older age (100-150 Myr) for AB Dor C -leading to closer agreement with the models than we find here.

suggested a slightly older age of 70-150 Myr. However, as Luhman et al. 2005 notes, the AB Dor moving group is systematically over-luminous compared to the Pleiades (age 100-120 Myr) by 0-0.1 magnitudes in $\mathrm{M}_{K s}$ vs. V-Ks plots (see their Figure 1).

We have found in Nielsen et al. 2005 similar results with a near-infrared color magnitude diagram, as seen in Figure 3. While the two groups of stars appear similar for the early-type members (where the isochrones overlap), beyond a color of $\mathrm{J}-\mathrm{Ks} \sim 0.4$, the lower main sequence of the AB Dor Moving group appears to be above that of the Pleiades by about 0.15 magnitudes. We have run a series of simulations that suggest that only $\sim 10 \%$ of the time would a group of Pleiades aged stars appear 0.15 magnitudes above the single-star locus as is observed for all the AB Dor group members.

We note that a 0.15 magnitude offset from the Pleiades single star locus suggests a group age of $\sim 70$ Myr from the Lyon group's models (Baraffe et al. 1998; see fig 3). We adopt an average age of $70 \pm 30 \mathrm{Myr}$ with $1 \sigma$ error bars. Hence, there is a $\sim 10 \%$ chance that the AB Dor moving group is as old as the Pleiades based on these color-magnitude diagrams.

Luhman et al. 2005 conclude that their increase in the age of the system (from 50 to $120 \mathrm{Myr}$ ) implies that the luminosity is correctly predicted by the models. But, as we will see in Section 4.2, even if the luminosity is close to (but less) than the predicted value at an age of $120 \mathrm{Myr}$ and $0.09 \mathrm{M}_{\text {sun }}$, there is still an error in the temperature which leads to a significant underestimation of the mass.

\subsection{HR Diagram and Evolutionary Models}

In order to further compare our observations of $\mathrm{AB}$ Dor $\mathrm{C}$ with the theoretical models, we consider an HR diagram with our measured values and the DUSTY models. Using the adopted spectral type of M7 and the absolute Ks from Close et al. 2005, we can derive an effective temperature and bolometric luminosity for AB Dor C. We plot AB Dor C in such an HR diagram in Figure 4, along with the DUSTY tracks, AB Dor Ba/Bb, 


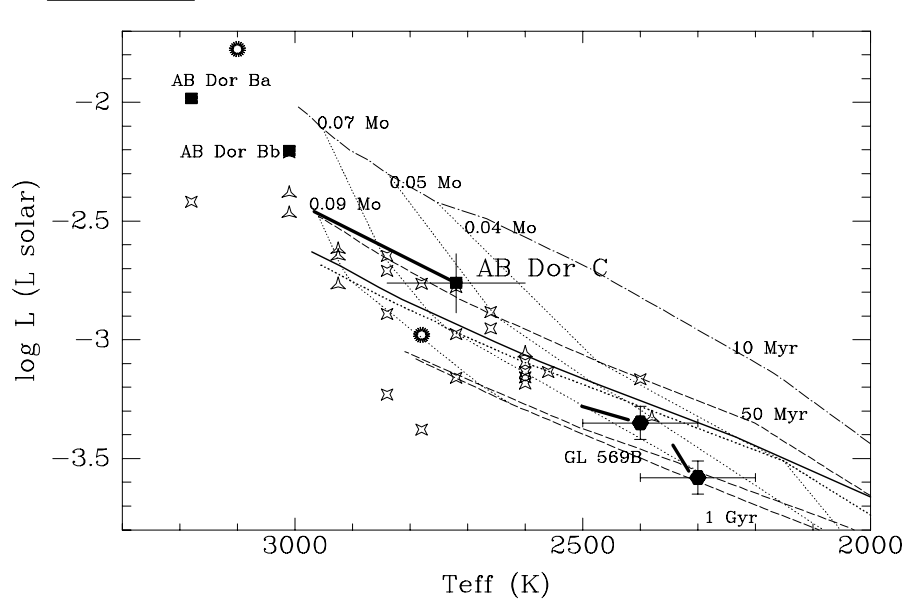

Figure 4. HR diagram showing low-mass Pleiades objects from Martín et al. 2000 (open stars), other low-mass members of the Pleiades taken from the literature (open triangles), and AB Dor $\mathrm{Ba} / \mathrm{Bb}$ (filled boxes). Both $\mathrm{AB}$ Dor $\mathrm{Ba} / \mathrm{Bb}$ and PPL $15 \mathrm{~A} / \mathrm{B}$ are shown both as individual objects and as a single, blended source (rings). The dotted vertical lines are iso-mass contours for the DUSTY models (from left to right, 0.09, 0.07,0.05, and $0.04 \mathrm{M}_{\text {sun }}$ ), while the more horizontal, dashed lines are the DUSTY isochrones (top to bottom, 10, 50, 100, 120, 500, $1000 \mathrm{Myr}$ ). Note that the DUSTY models predict a 70-100 Myr object of $0.09 \mathrm{M}_{\text {sun }}$ should be $\sim 300 \mathrm{~K}$ hotter than observed. From the location of $A B$ Dor $C$ on the HR diagram, one would derive a mass of $0.053 M_{\text {sun }}$, a factor of $\sim 1.7$ underestimate in mass. As the temperature and luminosities of the Pleiades objects in this plot were determined in the same manner used for $\mathrm{AB}$ Dor $\mathrm{C}$, and these Pleiades points mostly fall along the appropriate 120 Myr DUSTY isochrone, we are assured that our temperature scale and bolometric correction are reasonable (so these results cannot be improved by adopting another temperature scale since that would offset the Pleiades points from the $120 \mathrm{Myr}$ isochrone). With our $1 \sigma$ error bars, there is a $\sim 90 \%$ chance that the DUSTY models underestimate the mass of AB Dor $\mathrm{C}$ from the HR diagram.

and low-mass members of the Pleiades. We compile Pleiades members from Martín et al. 2000, as well as from other sources in the literature (Cluster identifications and spectral types from Briggs and Pye 2004, Pinfield et al. 2003, Terndrup et al. 1999, Festin 1998, Martín, Rebolo, and Zapatero-Osorio 1996, and Ks-band fluxes from the 2MASS catalog) The bolometric luminosities and temperatures for all these objects ( $\mathrm{AB}$ Dor $\mathrm{Ba} / \mathrm{Bb}, \mathrm{C}$, and the Pleiades members) are derived using Allen et al. 2003 and Luhman 1999 (dwarf scale), respectively.

As is seen in Figure 4, AB Dor C is overluminous, above the Pleiades sequence (as expected from a younger object, $\sim 70 \mathrm{Myr})$. We also show an thick line to its position in the HR diagram predicted by the DUSTY models appropriate to its age and $0.09 M_{\text {sun }}$ mass.

It has been suggested that this overluminosity could be explained if $\mathrm{AB}$ Dor $\mathrm{C}$ were a close, unresolved binary (Martín private communication; Marois et al. 2005). Were this the case, AB Dor $\mathrm{C}$ would split into two points in Figure 4, and move downward (as $\mathrm{AB}$ Dor $\mathrm{Ba} / \mathrm{Bb}$ and PPL 15 do when deblended), appearing consistent with the Pleiades locus. While this interpretation cannot be currently ruled out it is unlikely. To be stable against the pull of AB Dor A ( $\sim 2$ AU distant) the suggested binary brown dwarf would have to be $<0.1$ AU in separation to remain bound. Based on the survey of Reid 2003 we estimate the likelihood to be $<5 \%$ that such a tightly bound brown dwarf would be found. Moreover, as we will see in Figure 5 that this trend is seen for other low mass objects 


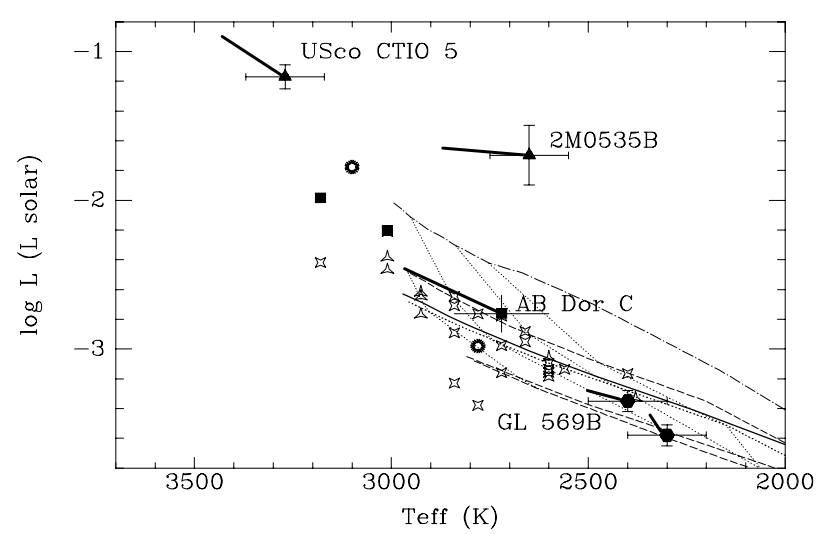

Figure 5. An expanded HR diagram with the Pleiades and the known low-mass (0.3-0.05 $\left.M_{\text {sun }}\right)$ with dynamical masses. The points with error bars mark objects with dynamical masses, with the diagonal lines representing the displacement from the measured luminosity and temperature to the values predicted by the DUSTY and Next-Gen models. Upper Sco CTIO 5 (Reiners et al. 2005; with the hottest "intermediate" temperature scale of Luhman et al. 1999), AB Dor C, 2MASS0535B (Stassun et al. 2005), and Gl 569 Ba/Bb (Zapatero Osorio et al. 2004) all show a systematic trend where the measured HR diagram location is cooler and fainter than the models' predictions. Seen another way, the masses predicted by the models are underestimates of the actual masses. This appears to be a general trend for all young low mass stars and high mass brown dwarfs. In the case of the older (300 Myr) Gl 569 B system, the offset is within the $1 \sigma$ uncertainties.

with dynamical masses as well - and they all cannot be spectroscopic binaries. Perhaps future very high spatial and spectral resolution observations will be able to investigate this possibility further.

Finally, we note an overall trend for young, low-mass objects where dynamic masses have been measured, as shown in Figure 5. There is a global offset to higher luminosities and temperatures for AB Dor C, USco CTIO 5, and Gl 569 Ba/Bb. These results suggest that there is a systematical trend of theoretical evolutionary tracks being somewhat too hot and slightly too luminous for measured dynamical masses in the range 0.3-0.05 $M_{\text {sun }}$ and ages $\sim 0.01-0.12$ Myrs.

\section{Acknowledgements}

We thank Gael Chauvin for providing an electronic copy of the spectrum of GSC 80470232, and Nadja Gorlova for providing spectra of many young, low-mass objects. We also thank the organizers of the IAUC 200 conference for the chance to present this work. LMC acknowledges support from the NASA Orgins and NSF CAREER programs that supported this work.

This publication makes use of data products from the Two micron All Sky Survey, which is a joint project of the University of Massachusetts and the Infrared Processing and Analysis Center/California Institute of Technology, funded by the National Aeronautics and Space Administration and the National Science Foundation.

\section{References}

Allen, P.R., Trilling, D.E., Korner, D.W., \& Reid, I.N.: 2003 ApJ 595, 1222

Baraffe, I., Chabrier, G., Allard, F., \& Hauschildt, P.H.: 1998 A\&A 337403

Burrows, A., Sudarsky, D., \& Lunine, J.I.: 2003 ApJ 569, 587 
Briggs, K.R. \& Pye, J.P.: 2004 MNRAS 353, 673

Chabrier, G., Baraffe, I., Allard, F., \& Hauschildt, P.H.: 2000 ApJ 542, 464

Chauvin, G., Lagrange, A.M., Lacombe, F., et al.: 2005 A\&A 430, 1027

Close, L.M., Lenzen, R., Guirado, J.C., et al.: 2005 Nature 433, 286

Cushing, M.C., Rayner, J.T., \& Vacca, W.D.: 2005 ApJ 623, 1115

Nielsen E.L., Close, L.M., Guirado, J.C., et al. 2005, Astron. Nachrichten, in press, Astroph/0509400

Festin, L.: 1998 MNRAS 2981, 34

Gorlova, N.I., Meyer, M.R., Rieke, G.H., \& Liebert, J.: 2003 ApJ 593, 1074

Guirado, J.C., Martí-Vidal, I., Marcaide, J.M., et al.: 2005 A\&A in press, astroph/0510199

Lenzen, R., Close, L., Brandner, W., Biller, B., \& Hartung, M.: 2004 SPIE 5492, 970

Lenzen, R., Hartung, M., Brandner, W., et al.: 2003 SPIE 4841, 944

Luhman, K.L.: 1999 ApJ 525, 466

Luhman, K.L., Stauffer, J.R. \& Mamajek, E.E.: 2005 ApJ 628, 69L

Luhman, K.L., \& Potter, D. 2006 ApJ, in press, astroph/0510666

Hillenbrand, L.A. \& White, R.J.: 2004 ApJ 604, 741

Maiolino, R., Rieke, G.H. \& Rieke, M.J.: 1996 AJ 111, 537

Marley, M.S., Fortney, J., Hubickyj, O., Saumon, D., et al. 2005 Presentation, 2005 Winter Conference on Astrophysics

Marois, C., Macintosh, B., Song, I., \& Barman, T.: 2005 astro-ph/0502382

Martín, E.L.: 2005 private communication

Martín, E.L., Brandner, W., Bouvier, et al.: 2000 ApJ 543, 299M

Martín, E.L., Rebolo, R., \& Zapatero-Osorio, M.R.: 1996 ApJ 469, 706M

Mohanty, S., Jayawardhana, R., \& Basri, G.: 2004 ApJ 609, 885M

Pinfield, D.J., Dobbie, P.D., Jameson, R.F., Steele, I.A., Jones, H.R.A. \& Katsiyannis, A.C.: 2003 MNRAS 342, 1241

Reiners, A., Basri, G., \& Mohanty, S.: 2005 accepted to ApJ, astro-ph/0506501

Stassun K. G. et al. 2005, Protostars \& Planets V conf. abstract

Terndrup, D.M., Krishnamurthi, A., Pinsonneault, M.H., \& Stauffer, J.R.: 1999 AJ 118, 1814

Zapatero Osorio, M. R., Lane, B.F., Pavlenko, Ya., Martín, E.L., Britton, M., \& Kulkarni, S.R.: 2004 ApJ 615, 958

Zuckerman, B., Song, I., \& Bessell, M.S.: 2004 ApJ 613L, 65

\section{Discussion}

ERIC GAIDOs: Then is your conclusion that the [dusty] evolutionary models are too hot for a given mass?

LAIRD Close: Yes, we find that in the case of AB Dor $\mathrm{C}$ the observed effective temperature appears lower than would be predicted by the models for a young $0.09 \mathrm{M}_{\text {sun }}$ companion. Or in other words the HR diagram would have predicted a lower mass from the dusty models given our observed spectral type and luminosity. A similar trend has been recently reported for the low mass binary star Upper Sco CTIO 5 system by Reiners et al.

RALPh Neuhauser: The gravity analysis of Monhanty et al. agrees with you that lowmass stars and high mass brown dwarfs will be underestimated in mass by these models

LAIRD Close: Yes

RAlPh Neuhauser: But they also suggest that the masses for very low mass brown dwarfs maybe over-estimated by the same models.

LAIRD ClOSE: Indeed, but we cannot confirm this prediction for exoplanets with our 
L. M. Close et al.

data since $\mathrm{AB}$ Dor $\mathrm{C}$ is too massive. It is interesting to note that the eclipsing binary brown dwarf in Orion (2MASS0535; Stassun et al. PPV abs.) may show just such a trend comparing its $\sim 0.035$ and $\sim 0.55 \mathrm{M}_{\text {sun }}$ components to the models. 